\title{
ON MATRIX COEFFICIENTS OF THE REFLECTION REPRESENTATION
}

\author{
J. MATTHEW DOUGLASS AND BRAD SHELTON
}

(Communicated by Warren J. Wong)

\begin{abstract}
We answer in the affirmative a question of Lusztig on the signs of certain matrix coefficients for the reflection representation of the Hecke algebra of a finite Weyl group. In the action of the Weyl group on the associated root system, the coefficients of the action of an element on a root are all of the same sign. It is shown that an appropriate generalization of this property holds for the reflection representation.
\end{abstract}

1.1 Let $(\mathscr{W}, S)$ be an indecomposable Coxeter pair where $\mathscr{W}$ is a finite Weyl group. Denote the Bruhat order on $\mathscr{W}$ by $<$ where $e$ is minimal and let $l(\cdot)$ be the length function on $\mathscr{W}$. Let $\mathscr{H}$ be the Hecke algebra of $\mathscr{W}$ over the polynomial ring $\mathbf{Q}\left[u^{1 / 2}, u^{-1 / 2}\right]$ (cf. [10]). Following Kazhdan and Lusztig [10], we have two standard bases of $\mathscr{H},\left\{T_{w} \mid w \in \mathscr{W}\right\}$ and $\left\{C_{w} \mid w \in \mathscr{W}\right\}$. Let $\Delta$ be a root system associated to $\mathscr{W}$ and for $s$ in $S$, let $\alpha_{s}$ be the corresponding simple root. Then the reflection representation $(\pi, E)$ of $\mathscr{H}$ has basis $\left\{d_{t} \mid t \in S\right\}$ and satisfies

$$
\pi\left(T_{s}\right) d_{t}=\left\{\begin{array}{ll}
-d_{s} & \text { if } s=t, \\
u d_{t}-u^{1 / 2}\left\langle\alpha_{t}, \alpha_{s}\right\rangle d_{s} & \text { if } s \neq t,
\end{array} \quad s, t \in S .\right.
$$

We refer the reader to [5] for the general properties of this representation. For $x$ in $\mathscr{W}$ and $s$ and $t$ in $S$, let $\pi(x, s, t)$ be the $t, s$-matrix entry of $\pi\left(T_{x}\right)$, that is

$$
\pi\left(T_{x}\right) d_{s}=\sum_{t \in S} \pi(x, s, t) d_{t} .
$$

Theorem 1.1. For $x$ in $\mathscr{W}$ and $s$ in $S$, the coefficients of the matrix entries $\pi(x, s, t), t \in S$, are all of the same sign as $l(x s)-l(x)$.

This theorem has been established previously in the case of $A_{n}$ by Lusztig and in the other classical cases by Tiwari [12]. Our proof does not rely on a case by case argument and works for the exceptional systems as well as the classical.

1.2 Let $\sim_{L}, \sim_{R}$ and $\sim_{L R}$ be the left, right and two-sided equivalence relations on $\mathscr{W}$ defined in [10]. The equivalence classes of their relations are

Received by the editors February 1, 1988 and, in revised form, March 14, 1988.

1980 Mathematics Subject Classification (1985 Revision). Primary 20C30. 
referred to, respectively, as left, right and two-sided cells. Let $\Gamma_{s}$ be the left cell containing the simple reflection $s$ and for $s$ and $t$ in $S$ put $\Delta_{s, t}=\Gamma_{s} \cap\left(\Gamma_{t}\right)^{-1}$. For $x$ in $\mathscr{W}$, define the matrix coefficients $h(x, s, t)$ by

$$
\pi\left(C_{x}\right) d_{s}=\sum_{t \in S} h(x, s, t) d_{t} .
$$

Lemma 1.2. For $s$ and $t$ in $S$ and $w$ in $\Delta_{s, t}$ there is a positive integer $n_{w, s, t}$ so that $h(w, s, t)=-n_{w, s, t}\left(u^{-1 / 2}+u^{1 / 2}\right)$.

The proof of this lemma can be found in [6]. It is a straightforward computation based on the formula

$$
\pi\left(C_{s}\right) d_{t}=\left\{\begin{array}{ll}
-\left(u^{1 / 2}+u^{-1 / 2}\right) d_{s} & \text { if } s=t, \\
-\left\langle\alpha_{t}, \alpha_{s}\right\rangle d_{s} & \text { if } s \neq t,
\end{array} \quad s, t \in S\right.
$$

Let $w_{0}$ be the longest element of $\mathscr{W}$. For $x$ and $y$ in $\mathscr{W}$ let $P_{x, y}(u)$ be the Kazhdan-Lusztig polynomial as defined in [10]. As usual, the polynomials $Q_{x, y}$ are defined by $Q_{x, y}=P_{w_{0} y, w_{0} x}$. We will use the notations

$$
u_{x}=u^{l(x)} \text { and } c(x, s)=\left\{\begin{array}{ll}
1 & \text { if } x>x s, \\
0 & \text { if } x<x s,
\end{array} \quad x \in \mathscr{W}, s \in S .\right.
$$

Also we will denote by $q \rightarrow \bar{q}$ the involution on $\mathbf{Q}\left[u^{1 / 2}, u^{-1 / 2}\right]$ given by $\overline{u^{1 / 2}}=$ $u^{-1 / 2}$.

Theorem 1.2. For $x$ in $\mathscr{W}$ and $s$ and $t$ in $S$,

$$
\pi(x, s, t)=u_{x}\left[\overline{\sum_{w \in \Delta_{s} . t} n_{w, s, t} u_{u s}^{1 / 2} u^{c(x, s)}\left(Q_{w, x s}(u)-Q_{w, x}(u)\right)}\right] .
$$

The proof of this theorem can also be found in [6]. It is based on a characterization of the two-sided cell $\mathbf{X}$ which corresponds to the reflection representation. This correspondence is described in [11]. In particular, it is shown in [6] that $\mathbf{X}$ is the two sided cell of $\mathscr{W}$ which contains all of the simple reflections and that $\mathbf{X}$ consists of exactly the nonidentity elements of $\mathscr{W}$ which have a unique reduced expression. We remark that we have not included several references needed for the proof of Theorem 1.2 and the pertinent facts about $\mathbf{X}$. A complete list of references can be found in [6].

From Theorem 1.2 it is clear that Theorem 1.1 will be proved as soon as we establish that the coefficients of $Q_{u, x s}-Q_{w, x}$ are nonnegative whenever $s \in S$, $w, x \in \mathscr{W}$ and $x s>x$.

2.1 Let $\mathfrak{g}$ be a complex semisimple Lie algebra with a Cartan subalgebra $\mathfrak{h}$ so that the Weyl group of $(\mathfrak{g}, \mathfrak{h})$ is $\mathscr{W}$. Let $\Delta$ be the root system of $(\mathfrak{g}, \mathfrak{h})$ with positive system $\Delta^{+}$compatible with $S$. Let $\mathfrak{b}=\mathfrak{h} \oplus \mathfrak{n}$ be the corresponding Borel subalgebra and let $\rho$ be half the sum of the positive roots. For $x$ in $\mathscr{W}$ we denote by $M_{x}$ the $\mathfrak{g}$-Verma module with highest weight $-x \rho-\rho$. That is, $M_{x}$ is the $\mathfrak{g}$-module induced from the one-dimensional $\mathfrak{b}$-module determined 
by the weight $-x \rho-\rho$. Each $M_{x}$ has a unique irreducible quotient which we denote $L_{x}$.

For any simple module $A$ and finite length module $B,[B: A]$ denotes the multiplicity of $A$ in a composition series for $B$. Recall that the socle of a module $M, \operatorname{soc}^{1}(M)$, is the maximal semisimple submodule of $M$. Inductively, $\operatorname{soc}^{i}(M)$ is the pull back into $M$ of the socle of $M / \operatorname{soc}^{i-1}(M)$. Put $\operatorname{soc}_{i}(M)=\operatorname{soc}^{i}(M) / \operatorname{soc}^{i-1}(M)$. The Loewy length of $M, l l(M)$, is the smallest $i$ for which $\operatorname{soc}^{i}(M)=M$. From [4] (as well as other sources) we know that $l l\left(M_{x}\right)=l(x)+1$.

Theorem 2.1 (Irving, [8]). For $w$ and $x$ in $\mathscr{W}$,

$$
Q_{w, x}(u)=\sum_{k}\left[\operatorname{soc}_{1+l(w)+2 k}\left(M_{x}\right): L_{w}\right] u^{k} .
$$

Irving's proof of Theorem 2.1 is based on the fact (also proved in [8]) that the socle filtration of $M_{x}$ coincides with the radical filtration of $M_{x}$. We observe that a slightly different version of Theorem 2.1 was also obtained by Gabber and Joseph in [7]. In the Gabber-Joseph version the layers of the socle filtration of $M_{x}$ are replaced by the layers of the Jantzen filtration of $M_{x}$ (defined in [9]). This requires the use of the Jantzen filtration conjecture of which there is no published proof (a proof was announced by Beilinson in [1]). Irving's result 2.1 is a formal consequence of the Kazhdan-Lusztig conjectures as proved in [ 2 or 3]. Either way we now proceed to prove Theorem 1.1 using the hereditary property of the filtration. (In the case of the Jantzen filtration this is exactly the Jantzen conjecture.)

Suppose now that $x$ and $w$ are in $\mathscr{W}, s$ is in $S$ and $x s>x$. Then the coefficient of $u^{k}$ in $Q_{w, x s}(u)-Q_{w, x}(u)$ is exactly

$$
\left[\operatorname{soc}_{1+l(w)+2 k}\left(M_{x s}\right): L_{w}\right]-\left[\operatorname{soc}_{1+l(w)+2 k}\left(M_{x}\right): L_{w}\right] \text {. }
$$

However, $M_{x}$ is a submodule of $M_{x s}$ and the socle filtration is hereditary for submodules, i.e. $\operatorname{soc}^{j}\left(M_{x}\right)=\operatorname{soc}^{j}\left(M_{x s}\right) \cap M_{x}$ for all $j$. This shows that the coefficient of $u^{k}$ is nonnegative. By Theorem 1.2 this completes the proof of Theorem 1.1. (Note: the above argument was observed in [8] as a corollary to Theorem 1.3. We include the argument for the sake of completeness.)

\section{REFERENCES}

1. A. Beilinson, Localization of representations of reductive Lie algebras, Proc. Internat. Cong. Math., North-Holland, 1983, pp. 699-710.

2. A. Beilinson and J. Bernstein, Localisation de g-modules, C. R. Acad. Sci. Paris 292 (1981), 15-18.

3. J. L. Brylinski and M. Kashiwara, Kazhdan-Lusztig conjecture and holonomic systems, Invent. Math. 64 (1981), 387-410.

4. D. Collingwood, R. Irving and B. Shelton, Filtrations on generalized Verma modules for Hermitian symmetric pairs, J. Reine Angew. Math. (to appear). 
5. C. Curtis, N. Iwahori and R. Kilmoyer, Hecke algebras and characters of parabolic type of finite groups with $(B, N)$-pairs, Inst. Hautes Études Sci. Publ. Math. 40 (1971), 81-116.

6. J. M. Douglass, Cells in Weyl groups corresponding to the reflection representation, preprint.

7. O. Gabber and A. Joseph, Towards the Kazhdan-Lusztig conjecture, Ann. Sci. École Norm. Sup. 14 (1981), 261-302.

8. R. Irving, The socle filtration of a Verma module, preprint.

9. J. Jantzen, Moduln mit einem Hochsten Gewicht, Lecture Notels in Math., vol. 750, SpringerVerlag, Berlin and New York, 1980.

10. D. Kazhdan and G. Lusztig, Representations of Coxeter groups and Hecke algebras, Invent. Math. 53 (1979), 165-184.

11. G. Lusztig, Unipotent characters of the symplectic and odd orthogonal groups over a finite field, Invent. Math. 64 (1981), 263-296.

12. H. Tiwari, Reflection representations of Hecke algebras of certain Weyl groups, preprint.

Department of Mathematics, University of Oregon, Eugene, Oregon 97403 